\title{
Comment
}

Neuroepidemiology 2010;35:214

DOI: $\underline{10.1159 / 000316647}$

\section{Delayed Start, Rapid Solution?}

\section{Amos D. Korczyn, Veronika Vakhapova}

Sackler Faculty of Medicine, Tel Aviv University,

Ramat Aviv, Israel

Neurodegenerative diseases, particularly Alzheimer's disease and Parkinson's disease (PD), are important targets for new therapies. The available drugs have a meager effect on cognition in Alzheimer's disease and an impressive beneficial effect on some motor dysfunctions in PD. However, these symptomatic treatments leave much to be desired, both in terms of non-responsive symptoms and disease progression. The difficulty of developing better therapies reflects our poor understanding of disease pathogenesis, the lack of appropriate animal models and the very slow progression of the diseases, which is also variable among patients. Thus in PD, the UPDRS motor examination scores decline at a rate of $8-9 \%$ per year in untreated patients [1]. In order to show that a novel drug reduces disease progression by $50 \%$ from a mean baseline UPDRS score of 20 (total) and 14 (motor part), several hundred patients need to be followed up for several months. However, the motor deterioration may lead to the institution of symptomatic therapy in some patients, causing obvious difficulties in assessing the degree of neuroprotection, unless the symptomatic drug is withdrawn for a period (which may be unethical or impractical). In addition, it is not clear for how long treatment should be withdrawn.

Usually, in a study which tries to assess a possible neuroprotective effect, the drug will be compared to placebo. If the drug has a symptomatic effect as well, the neuroprotective effect will be seen as a less steep progression of disability than in the placebo patients in the first phase of the study. It is quite common to put patients who have been assigned to the placebo group on the active drug after the first phase of the study has been completed ('delayed-on'). If these patients are followed up properly (and those who have been on active drug from the start will continue to receive the treatment), this will allow an even better demonstration of the neuroprotective effect. Theoretically, at least the patients originally assigned to placebo (and later to an active neuroprotective drug) should never reach as good a response as those who have been assigned to the neuroprotective drug from the start. In addition, this scheme will overcome the problem of a symptomatic effect because by the end of the study all patients will have received the active drug and any differences at that point should be due to a neuroprotective effect. This was the rationale behind the ADAGIO study of rasagiline in early PD [2].

The results of the ADAGIO study were positive, at least for the 1-mg rasagiline dose. However, as Vanacore [3] writes in this issue, there remain doubts on several points. The small effect size - 1.7 points on the UPDRS (and with a large SD of about 17 UPDRS points) - is of dubious clinical significance, particularly since it is not just the blinded motor score but also the ADL and the mental part of the UPDRS which are included in this benefit. The evaluation of the mental status and UPDRS is based on patients' qualitative reports and not on objective measurements.

Another concern is related to the fact that there is assumed linearity of the progression of the UPDRS over time. Because rasagiline has a symptomatic effect, patients who had entered the study on the placebo arm had a higher UPDRS score when they entered the second phase at the beginning of the active treatment, and therefore they are not really comparable to those who had started the active treatment 6 months earlier and had lower disability when entering the second phase.

All these considerations again teach us that there are no simple methods to prove modifying effects, particularly when the drug with the presumed neuroprotective influence also has a symptomatic effect, when the neuroprotective effect is small, and it does not stop disease progression completely.

\section{References}

1 Poewe WH, Wenning GK: The natural history of Parkinson's disease. Neurology 1996;47(suppl 3):S146-S152.

2 Olanow CW, Rascol O, Hauser R, Feigin PD, Jankovic J, Lang A, Langston W, Melamed E, Poewe W, Stocchi F, Tolosa E; ADAGIO Study Investigators: A double-blind, delayed-start trial of rasagiline in Parkinson's disease. N Engl J Med 2009;361:1268-1278.

- 3 Vanacore $\mathrm{N}$ : What is the clinical significance of the findings from the delayed-start trial of rasagiline in Parkinson's disease? Neuroepidemiology 2010;35:213.

Prof. Amos Korczyn, Sackler Faculty of Medicine

Tel Aviv University Medical School

Ramat Aviv 69978 (Israel)

Tel. +972 36974229, Fax +972 36409113

E-Mail amoskor@post.tau.ac.il

\section{KARGER}

Fax +41 613061234 E-Mail karger@karger.ch www.karger.com (c) 2010 S. Karger AG, Basel

0251-5350/10/0353-0214\$26.00/0 